\title{
Non-convulsive seizure related to Cremophor ELTM-free, polymeric micelle formulation of paclitaxel: a case report
}

\author{
Yoon Sun Shin, Kyung Jin Min, Seung Young Choi, Nak Woo Lee \\ Department of Obstetrics and Gynecology, Korea University Ansan Hospital, Korea University College of Medicine, Ansan, Korea
}

Paclitaxel is a chemotherapeutic agent that is effective against ovarian, breast, lung, and other cancers. Although peripheral neurotoxicity is among the most common side effects of paclitaxel treatment, central neurotoxicity is rarely reported. When centrally mediated side effects are observed, they are attributed to Cremophor EL ${ }^{\mathrm{TM}}$ (CrEL), a surfactant-containing vehicle used for paclitaxel administration. In the present report, we discuss the case of a 72-yearold woman with ovarian carcinoma who experienced a non-convulsive seizure following administration of a CrELfree, polymeric micelle formulation of paclitaxel. One week after her fourth round of chemotherapy, she experienced a transient episode of aphasia for 45 minutes. Electroencephalography demonstrated epileptiform discharges. To our knowledge, this is the first reported case of seizure associated with a CrEL-free formulation of paclitaxel. Although rare, patients and clinicians should remain aware of the risk of non-convulsive seizure following infusion of this paclitaxel formulation.

Keywords: Paclitaxel; Seizures; Neurotoxicity; Cremophor

\section{Introduction}

Neurological complications have been reported to occur in patients undergoing chemotherapy for cancer. Although the mechanisms underlying nervous system damage following chemotherapy remain unclear, neurotoxicity is a common side effect. Such damage may occur following the use of particular chemotherapeutic agents, or as an effect of dosage and route of administration [1]. Paclitaxel is a particularly effective antitumor drug, especially in cases of ovarian, breast, lung, and similar solid cancers. Peripheral neurotoxicity is common in patients undergoing treatment with paclitaxel; however, central nervous system (CNS) toxicity is only rarely reported. Cremophor EL ${ }^{\mathrm{TM}}$ (CrEL) is a surfactant-containing formulation vehicle used for various drugs with poor water-solubility, including paclitaxel. CrEL itself is known to be somewhat neurotoxic, and to antagonize paclitaxel-mediated cytotoxicity $[2,3]$. While reported CNS-mediated side effects of paclitaxel include generalized seizures and acute encephalopathy [4-6], such effects have been attributed to the use of CrEL-based formulations. In the present report, we discuss a case of nonconvulsive seizure occurring in a 72-year-old woman with ovarian carcinoma following administration of a standard dose of a CrEL-free, polymeric micelle formulation of paclitaxel (Genexol-PM) and carboplatin.

\section{Case report}

A 72-year-old woman was admitted to our Department of Gynecology in December 2015, due to a pelvic mass. The patient had no relevant medical history other than hypertension. Abdominal computed tomography (CT) revealed a large (9.2 $\mathrm{cm})$, heterogeneous mass at the superior-posterior aspect of

Received: 2017.05.31. Revised: 2017.08.07. Accepted: 2017.08.17. Corresponding author: Nak Woo Lee

Department of Obstetrics and Gynecology, Korea University Ansan Hospital, Korea University College of Medicine, 123 Jeokgeum-ro, Danwon-gu, Ansan 15355, Korea

E-mail: nwlee@korea.ac.kr

https://orcid.org/0000-0001-9405-7574

Articles published in Obstet Gynecol Sci are open-access, distributed under the terms of the Creative Commons Attribution Non-Commercial License (http://creativecommons. org/licenses/by-nc/3.0/) which permits unrestricted non-commercial use, distribution, and reproduction in any medium, provided the original work is properly cited.

Copyright () 2018 Korean Society of Obstetrics and Gynecology 


\title{
Obstetrics \& Gynecology Science
}

\author{
Vol. 61, No. 3, 2018
}

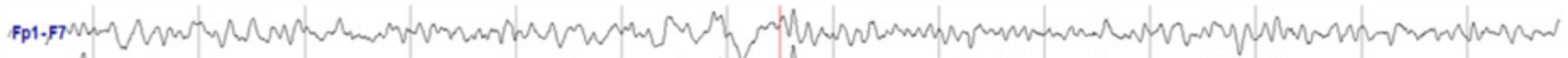

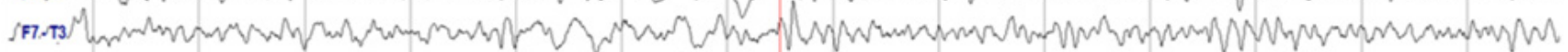

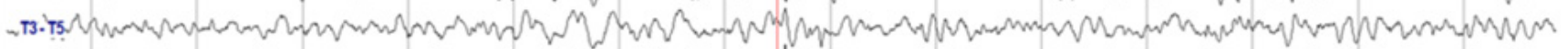

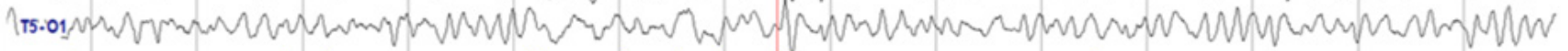

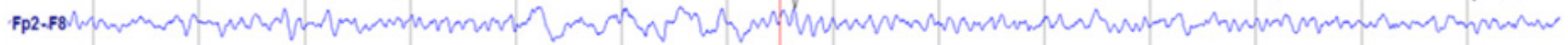
158.74.

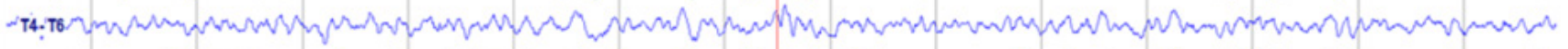

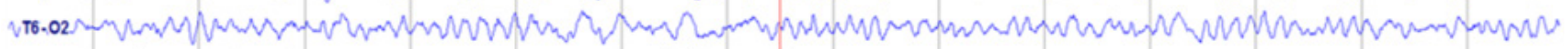

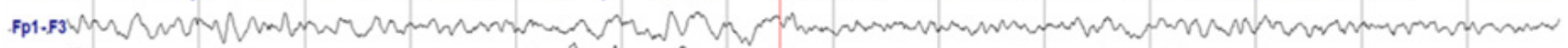

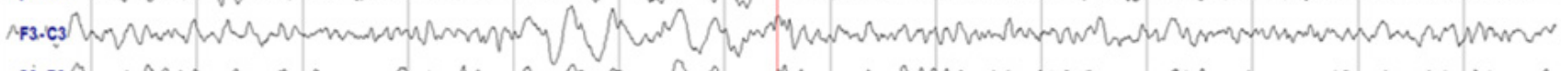

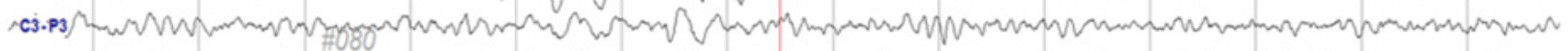

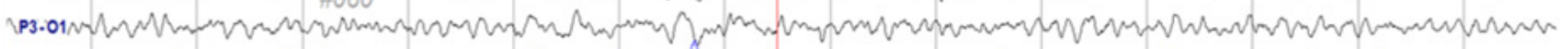

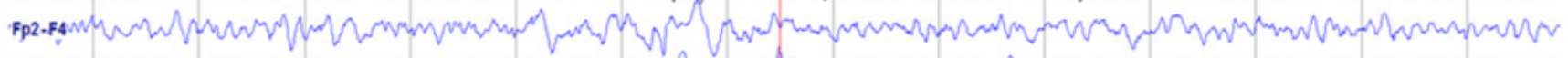

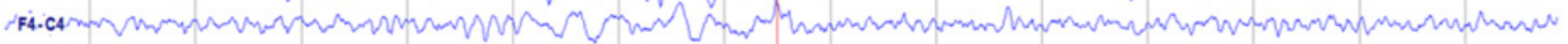

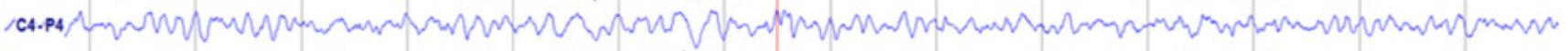

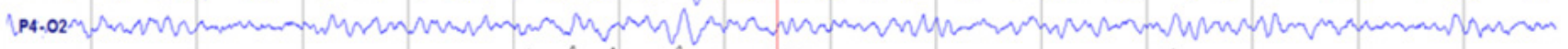

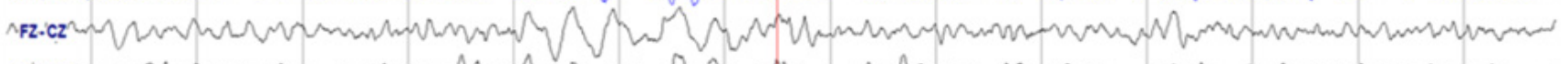

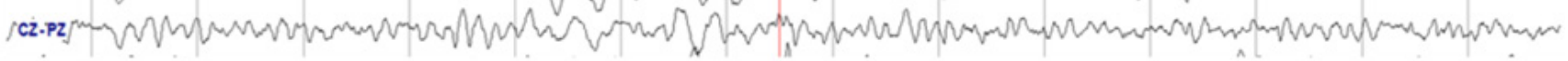

Fig. 1. Initial electroencephalogram showing frequent spikes arising from the left temporal area.

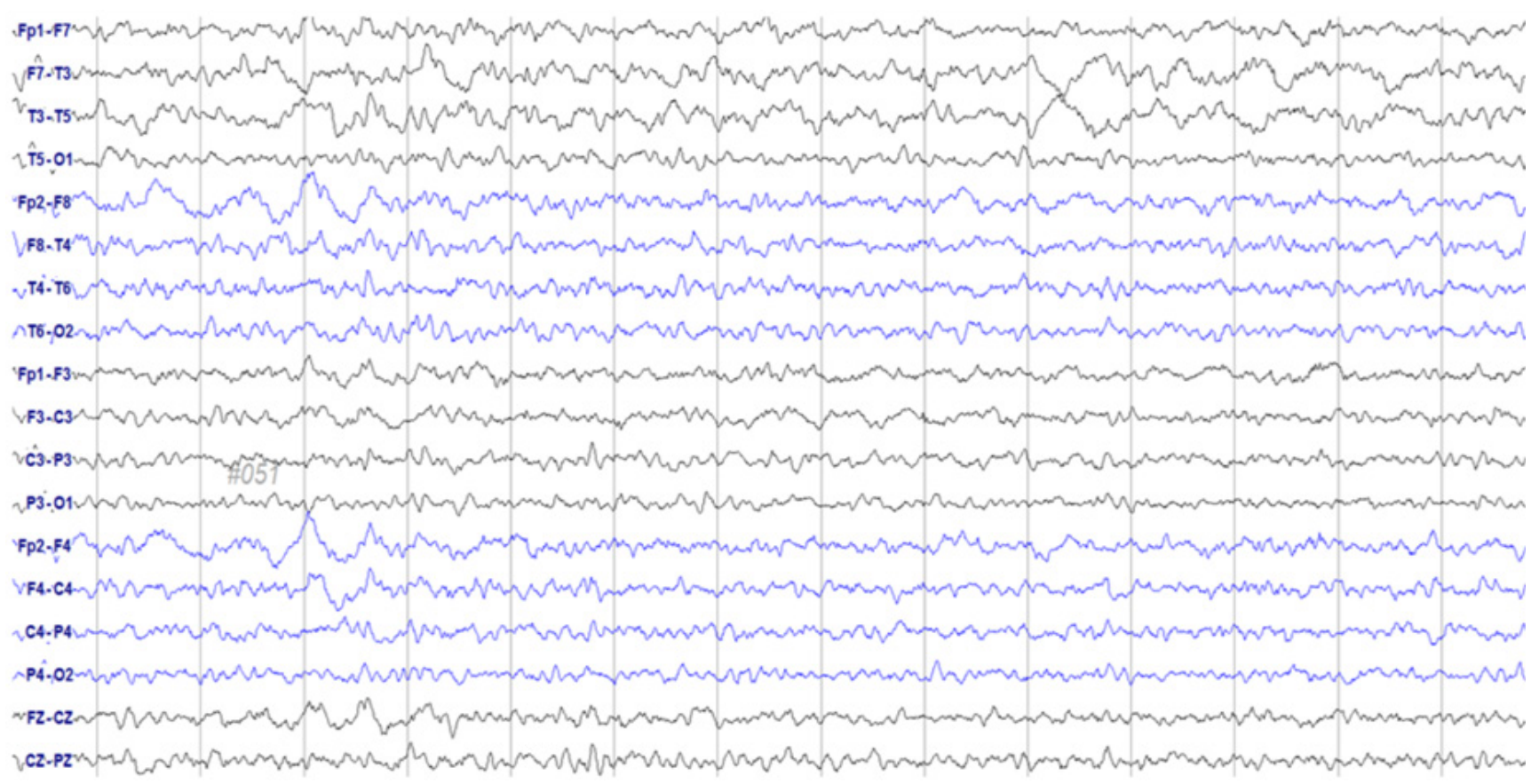

Fig. 2. Electroencephalogram performed 4 days following seizure and discontinuation of treatment, showing no epileptiform discharges.

the uterus. She underwent pelviscopic left salpingo-oophorectomy; histology confirmed the mass as a left ovary carcinosarcoma (a malignant Müllerian-mixed tumor). Adjuvant chemotherapy was initiated 1 month after surgery with paclitaxel and carboplatin, which were administered every 3 weeks. Following pretreatment with intravenous (IV) dexamethasone 20 mg 30 minutes, 6 and 12 hours before administration, IV lorazepam $2 \mathrm{mg}$, and ramosetron $0.3 \mathrm{mg} 1$ hour before admin- 


\section{Obstetrics \& Gynecology Science}

Yoon Sun Shin, et al. Non-convulsive seizure due to paclitaxel

istration, a conventional dose $\left(260 \mathrm{mg} / \mathrm{m}^{2} ; 457.6 \mathrm{~g}\right.$ based on a body surface area of $1.74 \mathrm{~m}^{2}$ ) of Genexol-PMTM - a novel CrEL-free, polymeric micelle formulation of paclitaxel — was administered. Chemotherapy was well-tolerated for 3 cycles, although the patient suddenly slipped and fell while waiting in the radiology department for her follow-up CT evaluation. She was mentally alert yet aphasic for 45 minutes. Her blood pressure was within the normal range, and laboratory results revealed no abnormalities. Brain magnetic resonance imaging revealed no focal signal intensities or structural lesions. An initial electroencephalogram (EEG) was obtained immediately, on which frequent spikes and intermittent slow-wave activity suggestive of a partial seizure disorder were identified; the focus appeared to be the left temporal area (Fig. 1). She was treated with an antiepileptic drug (levetiracetam $500 \mathrm{mg}$ twice/day), and no further abnormal activity was detected during her hospitalization. Her EEG normalized after 4 days (Fig. 2). Additional systemic chemotherapy was discontinued, and the patient did not experience any overt seizures during the following 3 months of follow-up observation.

\section{Discussion}

Paclitaxel is a potent chemotherapeutic agent against solid tumors that stabilizes and prevents the depolymerization of microtubules by targeting tubulin. Myelosuppression and peripheral neurotoxicity are frequently observed complications during paclitaxel treatment, while CNS toxicity upon IV administration is limited, presumably due to the blood-brain and blood-cerebrospinal fluid barriers. However, several cases of CNS-mediated side effects including acute encephalopathy and seizures have been reported, suggesting that administration of conventional doses of paclitaxel may still induce CNS toxicity in the absence of brain metastasis or blood-brain barrier disruption [4-6].

Non-convulsive seizures can occur in a wide variety of conditions including cerebrovascular diseases, CNS tumors, medication-induced conditions, metabolic disturbances, and inflammatory/infectious diseases. Furthermore, various conditions have been reported to lower seizure thresholds. Although symptoms may not be specific, suspected clinical diagnoses should be confirmed via EEG [7].

In the present case, the patient underwent baseline assessment prior to chemotherapy administration, during which she reported no history of alterations in mental status or seizures. Further evaluation revealed no signs of head injury, infection, or damage to the brain. Her only medication at the time was an antihypertensive agent. Laboratory tests including complete blood count, metabolic panel, blood glucose, and urine analysis performed following the seizure indicated no abnormal findings. Furthermore, full neurologic examination and brain imaging revealed no abnormalities, and the patient exhibited no additional neurological symptoms or EEG abnormalities following discontinuation of chemotherapy. Based on this clinical information, other factors were ruled out, and the chemotherapy agent was regarded as the most probable etiology of the patient's seizure.

To our knowledge, this is only the second reported case of a non-convulsive seizure secondary to treatment with paclitaxel. However, our patient was treated with CrEL-free paclitaxel. The toxic surfactant CrEL has been regarded as the major cause of seizures in previous case reports [3-6], indicating that this is the first reported case of seizure associated with a CrELfree formulation of paclitaxel (Genexol-PMTM). This formulation of paclitaxel is less toxic than CrEL-based formulations, supporting the assumption that paclitaxel itself can induce CNS symptoms. However, the patient's other medications — including the antiemetic, steroid, and carboplatin — may have also have contributed to her CNS involvement.

A recent open-label, phase 1 trial [8] was conducted to determine the maximum tolerated dose of Genexol-PM ${ }^{\mathrm{TM}}$ when combined with carboplatin as a first-line treatment for advanced ovarian cancer. Dose-limiting toxicities were not reported at $220 \mathrm{mg} / \mathrm{m}^{2}$ or $260 \mathrm{mg} / \mathrm{m}^{2}$, although one patient reported general pain (grade 3, based on National Cancer Institute CTCAE v3.0) at a dose of $300 \mathrm{mg} / \mathrm{m}^{2}$. Most patients in the trial experienced adverse events, including mild peripheral neuropathy; however, neither the frequency nor severity of these events differed significantly among dose levels. The majority of patients also recovered without sequelae, and there were no reported instances of CNS neurotoxicity. This previous study recommended a dose of $300 \mathrm{mg} / \mathrm{m}^{2}$ or less during phase II trials.

In the present case, the patient received $260 \mathrm{mg} / \mathrm{m}^{2}$ of Genexol-PM ${ }^{\mathrm{TM}}$ once every 3 weeks. This dose was assumed to be below the neurotoxic threshold of paclitaxel [8,9]. However, 4 rounds of chemotherapy were performed prior to seizure occurrence, resulting in a cumulative dose of $1,040 \mathrm{mg} / \mathrm{m}^{2}$. Although the safe range of cumulative paclitaxel dosage has 


\title{
Obstetrics \& Gynecology Science
}

\author{
Vol. 61, No. 3, 2018
}

not been identified, paclitaxel neurotoxicity is known to be dose-dependent, and increasing cumulative doses of paclitaxel have been associated with a greater risk of neurotoxicity $[10,11]$. Furthermore, because the patient's seizure occurred 1 week after the administration of paclitaxel, we believe her case may have been associated with CNS neurotoxicity due to the cumulative dose of paclitaxel. In accordance with this assumption, several studies have reported delayed seizures or encephalopathy following paclitaxel administration in the absence of other underlying medical factors $[5,6]$.

The putative association between the use of Genexol-PM ${ }^{\mathrm{TM}}$ and the delayed non-convulsive seizure in the patient of the present case was ranked as probable on the Naranjo Adverse Drug Reaction Probability Scale (score: 7) [12]. Thus, it is likely that this late-onset, non-convulsive seizure occurred due to administration of paclitaxel itself, rather than the vehicle formulation chosen. Initial evaluation of the patient's medical condition and other possible etiologies is recommended when patients present with neurologic symptoms. When other factors have been ruled out, patients and clinicians should remain aware of the rare yet potential risk of non-convulsive seizures following infusion of CrEL-free paclitaxel.

\section{Conflict of interest}

No potential conflict of interest relevant to this article was reported.

\section{References}

1. Magge RS, DeAngelis LM. The double-edged sword: neurotoxicity of chemotherapy. Blood Rev 2015;29:93100.

2. Gelderblom H, Verweij J, Nooter K, Sparreboom A. Cremophor EL: the drawbacks and advantages of vehicle selection for drug formulation. Eur J Cancer 2001;37:1590-8.
3. Deepa G, Ashwanikumar N, Pillai JJ, Kumar GS. Polymer nanoparticles--a novel strategy for administration of Paclitaxel in cancer chemotherapy. Curr Med Chem 2012;19:6207-13.

4. Illán-Gala I, Díaz de Terán FJ, Alonso P, Aguilar-Amat MJ. Nonconvulsive status epilepticus secondary to paclitaxel administration. Epilepsy Behav Case Rep 2015;4:20-2.

5. O'Connor TL, Kossoff E. Delayed seizure associated with paclitaxel-Cremophor el in a patient with early-stage breast cancer. Pharmacotherapy 2009;29:993-6.

6. Muallaoğlu S, Koçer M, Güler N. Acute transient encephalopathy after weekly paclitaxel infusion. Med Oncol 2012;29:1297-9.

7. Beleza P, Rocha J, Pinho J. Diagnosis, etiology, and treatment of nonconvulsive status epilepticus, a semiological oriented review. Neurologist 2015;19:160-7.

8. Lee SW, Kim YM, Kim YT, Kang SB. An open-label, multicenter, phase I trial of a cremophor-free, polymeric micelle formulation of paclitaxel combined with carboplatin as a first-line treatment for advanced ovarian cancer: a Korean Gynecologic Oncology Group study (KGOG3016). J Gynecol Oncol 2017;28:e26.

9. Kim TY, Kim DW, Chung JY, Shin SG, Kim SC, Heo DS, et al. Phase I and pharmacokinetic study of Genexol-PM, a cremophor-free, polymeric micelle-formulated paclitaxel, in patients with advanced malignancies. Clin Cancer Res 2004; 10:3708-16.

10. Park SB, Goldstein D, Krishnan AV, Lin CS, Friedlander $M L$, Cassidy J, et al. Chemotherapy-induced peripheral neurotoxicity: a critical analysis. CA Cancer J Clin 2013;63:419-37.

11. van Gerven JM, Moll JW, van den Bent MJ, Bontenbal M, van der Burg ME, Verweij J, et al. Paclitaxel (Taxol) induces cumulative mild neurotoxicity. Eur J Cancer 1994;30A:1074-7.

12. Naranjo CA, Busto U, Sellers EM, Sandor P, Ruiz I, Roberts $E A$, et al. A method for estimating the probability of adverse drug reactions. Clin Pharmacol Ther 1981;30:239-45. 